\title{
Microstructure and transport properties of multiwall carbon nanotube-reinforced barium zirconium titanate ceramics
}

\author{
RAGHAVENDRA SAGAR ${ }^{1,2}$ and R L RAIBAGKAR ${ }^{3, *}$ \\ ${ }^{1}$ Department of Post Graduate Studies and Research in Materials Science, Gulbarga University, Gulbarga 585 106, \\ Karnataka, India \\ ${ }^{2}$ Department of Physics, Mangalore Institute of Technology \& Engineering, Badaga Mijar, Moodabidre, Dakshina \\ Kannada 574 225, Karnataka, India \\ ${ }^{3}$ Department of Post Graduate Studies and Research in Applied Electronics, Gulbarga University, Gulbarga 585106 , \\ Karnataka, India \\ *Author for correspondence (rlraibagkar@ rediffmail.com)
}

MS received 29 May 2018; accepted 10 March 2019; published online 21 May 2019

\begin{abstract}
The influence of multiwall carbon nanotubes (MWCNTs) reinforced on microstructures and their transport properties on pure and cerium-substituted barium zirconium titanate (BZT) ceramics are reported in this study. The MWCNTs were prepared by a low-temperature sonochemical/hydrothermal method using dichloromethane as a carbon source. These MWCNTs were mixed with the as-prepared ceramic powders before heat treatment to obtain a reinforced product. The scanning electron micrographs reveal the successful incorporation of carbon nanotubes in BZT ceramics. The temperaturedependent direct current (dc)-resistivity was less for MWCNT-reinforced ceramics in contrast to that of pure ceramics. The decrease in the dc resistivity was due to the superior electrical behaviour of MWCNTs, which act as a connector between ceramic grains. The Seebeck coefficient of cerium-substituted-BZT improved after reinforcing the MWCNTs.
\end{abstract}

Keywords. Multiwall carbon nanotubes; barium zirconium titanate; microstructure; dc-conductivity; Seebeck coefficient.

\section{Introduction}

After successful investigation of various rare earth ion-substituted barium zirconium titanate (BZT) samples in the form of $\left(\mathrm{Ba}_{1-x} \mathrm{Re}_{x}\right)\left(\mathrm{Zr}_{0.52} \mathrm{Ti}_{0.48}\right) \mathrm{O}_{3}(\mathrm{Re}=\mathrm{Gd}, \mathrm{Sm}, \mathrm{Ce}, \mathrm{Pr}$, $\mathrm{Ho}$ and $\mathrm{Nd}$ ) in our previous studies, while in this paper we report the influence of multiwall carbon nanotube (MWCNT) reinforcement in BZT ceramics. MWCNTs being a polymorphic form of carbon have fascinated many researchers due to their excellent mechanical, electrical, thermal and chemical properties [1-4]. On the other hand, BZT is an important material for many electronic device applications due to its tunable electrical and dielectric performance unlike chemically and thermally unstable strontium and lead-based perovskites [5-8]. However, the extreme insulating behaviour of BZT at room temperature limits its extensive use in various applications only in thermistors and dielectric resonators [9]. Hence, reinforcing a conducting phase can be a better initiative to improve the transport properties of BZT ceramics. Thus, carbon nanotubes (CNTs) can be considered as a promising constituent for reinforcing in the BZT matrix, which offers high-electrical performances unattainable from host BZT ceramics.

CNT-reinforced ceramics have become fascinating materials for intense research due to their exciting structural configurations. Most of the attempts have been made to improve the mechanical properties of composites through incorporating CNTs [10]. Also, many researchers reported the improved electrical behaviour with different volume fractions of MWCNTs reinforced in the ceramic matrix. The electrical resistivity from 0.1449 to $0.2752 \Omega \mathrm{cm}$ of $\mathrm{MWCNT} / \mathrm{BaTiO}_{3}$ nanocomposite ceramics was found to enlarge progressively as the amount of MWCNT added increased from 0 to $3 \mathrm{wt} \%$ [11]. Martin Michalek et al [12] reported the enhancement of electrical conductivity from $10^{-12} \mathrm{~S} \mathrm{~m}^{-1}$ in the pure alumina to $2.7 \times 10^{-1} \mathrm{~S} \mathrm{~m}^{-1}$ in the composites containing $2 \mathrm{vol} \%$ of MWCNTs. MWCNTs were used effectively to convert electrically insulating alumina into electrically conducting composites and follow a power law of percolation with a low value of percolation threshold of $0.45 \mathrm{wt} \%$ [13]. The reported experimental result shows that the electrical conductivity of the composites increases with the CNT contents and decreases in the range of the conductivity of semiconductors. The nanocomposite with the highest CNT content has an electrical conductivity of $3336 \mathrm{~S} \mathrm{~m}^{-1}$ at near room temperature, which is about 13 orders of magnitude increase over that of pure alumina [14]. The direct current (dc) conductivity $\left(\sigma_{\mathrm{dc}}\right)$ of MWCNT addition in $3 \mathrm{~mol} \%$ yttria-stabilized tetragonal polycrystalline zirconia composites demonstrated a typical percolation behaviour with a rule, $\ln \sigma_{\mathrm{dc}} \propto f^{-1 / 3}$, where $f$ is the weight fraction of the conducting component showing tunnelling conduction responsible for the lower 
percolation threshold $[15,16]$. The increase in the electrical conductivity after reinforcement in ceramics is mainly due to the electrical percolation because of the enormous aspect ratio $(1000-10,000)$ of the CNTs [17]. On the other hand, effective dispersion and distribution of CNTs in the ceramic matrix always remains a challenge. The effective dispersion of CNTs in the ceramic matrix is the single most important parameter governing the transfer of CNT properties to the matrix material [18]. This is most suitable with regard to the formation of an electrically conducting CNT ceramic network. The homogeneous dispersion of CNTs and tight interface bonding are important challenges in reinforcing MWCNTs into the ceramic matrix because of the incompatibility between the electrovalent bond of inorganic ceramic materials and the covalent bond of $\mathrm{sp}^{2}$-bonded CNTs [19].

After extensive literature review and the analysis of the importance of CNT-reinforced electroceramics in many applications, we performed our studies to improve their electrical behaviour by reinforcing MWCNTs into the $\left(\mathrm{Ba}_{1-x} \mathrm{Re}_{x}\right)$ $\left(\mathrm{Zr}_{0.52} \mathrm{Ti}_{0.48}\right) \mathrm{O}_{3}$ ceramics [20-26]. The ceramic samples with the chemical composition $\left(\mathrm{Ba}_{1-x} \mathrm{Ce}_{x}\right)\left(\mathrm{Zr}_{0.52} \mathrm{Ti}_{0.48}\right) \mathrm{O}_{3}$ (BCZT) where $x=0,0.1$ and 0.2 were selected as ceramic matrices due to their better electrical behaviour. In this paper, the MWCNTs prepared by using a low-temperature sonochemical hydrothermal technique were reinforced into BCZT ceramics. The microstructure and electrical properties of CNT-reinforced pure and cerium-doped BZT ceramics have been investigated and compared here with our earlier reports.

\section{Experimental}

\subsection{Preparation of MWCNTs}

The MWCNTs were prepared by a well-known sonochemical/hydrothermal route as reported by Manafi et al [27]. In a typical synthesis route, dichloromethane $\left(\mathrm{CH}_{2} \mathrm{Cl}_{2}\right)$ was used as a carbon source to obtain MWCNTs. In this route, $40 \mathrm{ml}$ of $\mathrm{CH}_{2} \mathrm{Cl}_{2}, 1 \mathrm{~g}$ of lithium and $1 \mathrm{~g}$ of cobalt chloride $\left(\mathrm{CoCl}_{2}\right)$ were dissolved in $15 \mathrm{ml}$ of $\mathrm{NaOH}$ solution $\left(5 \mathrm{~mol}^{-1}\right)$ under an ultrasonic bath for $0.5 \mathrm{~h}$ at ambient temperature. Then, this aqueous solution was transferred to a Teflon-lined stainless steel autoclave. The hydrothermal synthesis was carried out at $180^{\circ} \mathrm{C}$ for $24 \mathrm{~h}$ in an electric oven. After the reaction, the obtained black powder was successively washed with ethanol, dilute hydrochloric acid and distilled water to remove residual impurities, such as amorphous carbon and catalyst and then dried at $110^{\circ} \mathrm{C}$ for $24 \mathrm{~h}$.

\subsection{Preparation of CNT-reinforced ceramics}

The ceramic samples prepared by the solid state reaction with composition of $\left(\mathrm{Ba}_{1-x} \mathrm{Ce}_{x}\right)\left(\mathrm{Zr}_{0.52} \mathrm{Ti}_{0.48}\right) \mathrm{O}_{3}(x=$ $0.0\{\mathrm{BZT}\}, 0.1$ (BCZT10\} and $0.2\{\mathrm{BCZT} 20\}$ ) were used as ceramic samples along with the prepared MWCNTs as reinforcements. The detailed synthesis procedure of the ceramic

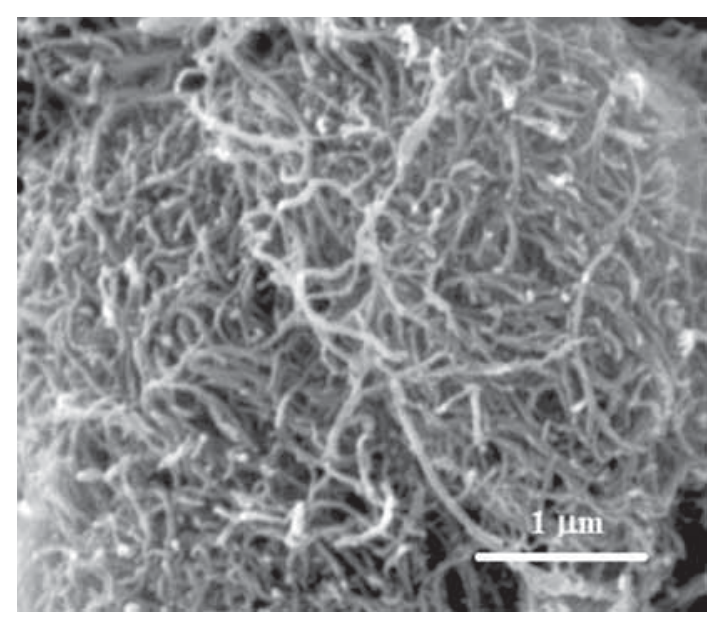

Figure 1. SEM image of MWCNTs prepared by the sonochemical/hydrothermal route.

samples is reported elsewhere [21]. The ceramic samples and $1 \%$ MWCNTs were weighed and then mixed using an agate mortar and pestle to obtain a homogeneous mixture. The obtained MWCNT/ceramic mixtures were pressed isostatically into circular pellets with a pressure of $100 \mathrm{MPa}$ using a hydraulic press and sintered at $500^{\circ} \mathrm{C}$ for $2 \mathrm{~h}$ using a temperature controlled programmable muffle furnace in air to obtain the final product.

\subsection{Characterization}

The microstructures of the MWCNT-reinforced ceramics were observed using a scanning electron microscope (model JSM/4048/SM, JEOL Japan). The grain size of the samples was evaluated by a line intercept method. For electrical characterization, MWCNT-reinforced ceramic pellets with dimensions (diameter of $10 \mathrm{~mm}$ and thickness of $2 \mathrm{~mm}$ ) were coated with silver paste to obtain uniform electrical measurements. The dc resistivity measurement was performed using a two probe technique in the temperature range of 323-773 K. The thermoelectric power (TEP) of the BCZT samples was calculated by adjusting the thermal gradient $(\Delta T)$ across the samples. The resulting thermal electro-motive force $(\Delta E)$ across the pellet was measured by using a digital microvoltmeter and the Seebeck coefficient $(S)$ was estimated.

\section{Results and discussion}

The microstructure of sonochemically/hydrothermally prepared MWCNTs is shown in figure 1. The product obtained is consistent with the micrographs reported by Manafi et al [27]. The MWCNTs were homogenously decorated with uniform distribution and linked with each other in the dense microstructure. These CNTs have a diameter ranging in $\mathrm{nm}$ and length in $\mu \mathrm{m}$. Also, we observe that the MWCNTs are sinuous, entangled and highly pure because of 


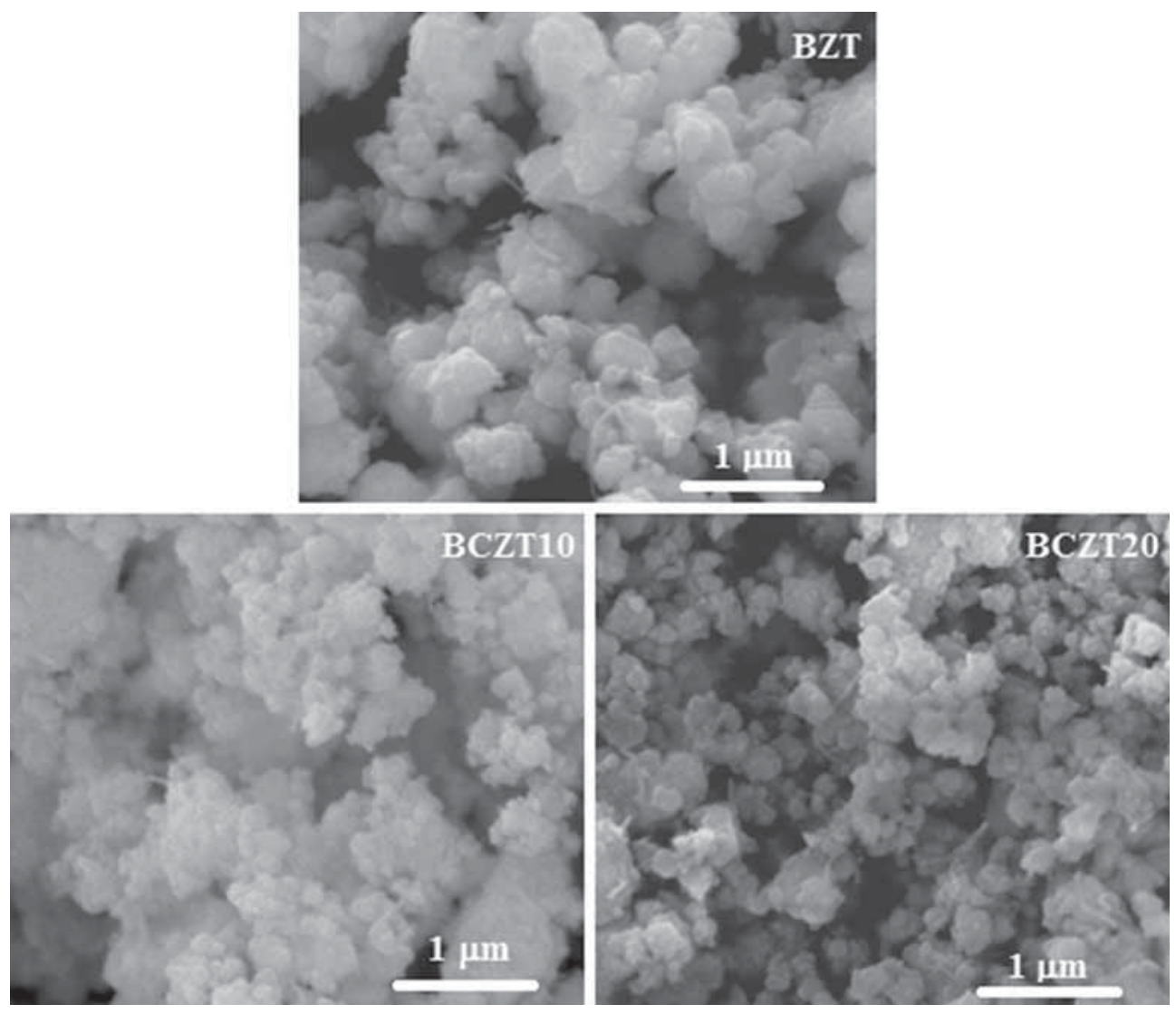

Figure 2. SEM images of MWCNT-reinforced BZT and BCZT ceramics.

successive purification from ethanol, dilute hydrochloric acid and distilled water.

Figure 2 shows the SEM image of CNT-reinforced BZT, BCZT10 and BCZT20 samples. From micrographs, we observe that the micrographs of all the samples show randomly distributed MWCNTs within the microstructure of homogenously distributed grains. The MWCNTs are mostly situated at grain boundaries with random orientation and surrounded by the other ceramic grains with a clean interface between MWCNTs and ceramic grains. We believe that the formation of MWCNT-reinforced ceramics is mainly due to the unique flexible nature of CNTs which makes them bend and pass through the space between ceramic powders. The porous microstructure was mostly due to sintering at lower temperature. Nevertheless, the lowsintering temperature is an important aspect to preserve the MWCNTs in the sintered composites [28]. Interestingly, the microstructure of MWCT embedded BCZT20 exhibited the smallest grain size of $\sim 108 \mathrm{~nm}$ as compared to $123 \mathrm{~nm}$ of BCZT10 and $146 \mathrm{~nm}$ of BZT. We assume that the MWCNTs substantially reduce the grain growth and pins down grain boundaries to reduce their mobility during creep.

The temperature dependent $\rho_{\mathrm{dc}}$ of the MWCNT-reinforced ceramics is shown in figure 3 . The results reveal that the

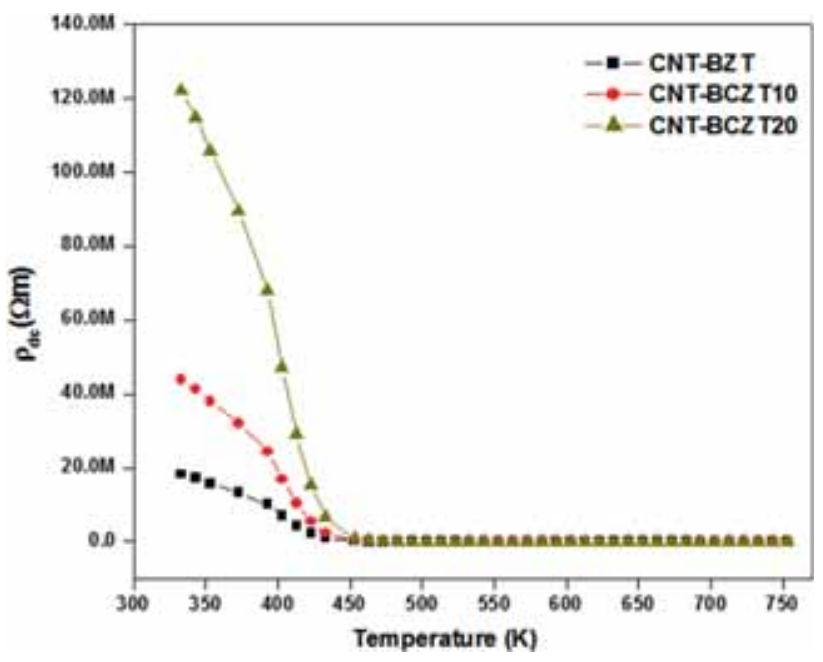

Figure 3. Temperature dependant $\rho_{\mathrm{dc}}$ of MWCNT-reinforced BZT and BCZT ceramics.

resistivity is highly dependent on the MWCNT reinforcement and cerium concentration in BZT. All CNT embedded ceramic samples exhibit highly insulating behaviour at room temperature and the magnitude of resistivity also increases 


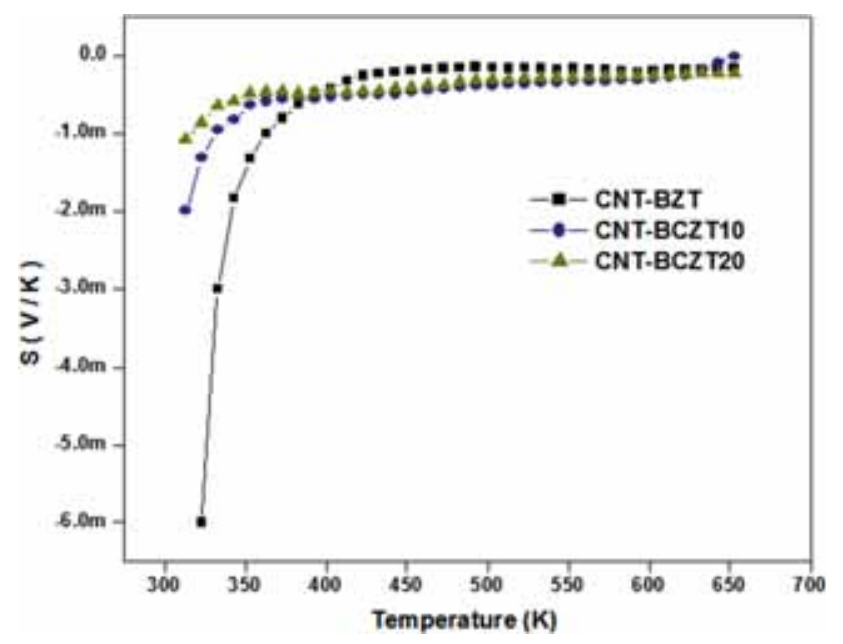

Figure 4. TEP measurement of MWCNT-reinforced BCZT ceramics.

as a function of cerium. At room temperature, even though the CNT embedded ceramics were insulative and exhibit $\rho_{\mathrm{dc}}$ around $10^{7}-10^{8} \Omega \mathrm{m}$, they were relatively less resistive compared to the BZT, BCZT10 and BCZT20, as reported in our earlier studies. After reinforcing MWCNTs in ceramics, the magnitude of resistivity drastically decreases from $37.85 \times 10^{6}$ to $20 \times 10^{6} \Omega \mathrm{m}$ in BZT, from $1.01 \times 10^{9}$ to $43 \times 10^{6} \Omega \mathrm{m}$ in BCZT10 and from $1.1 \times 10^{9}$ to $121 \times 10^{6} \Omega \mathrm{m}$ in BCZT20 [21] and the thermally activated temperature was in between 323 and $423 \mathrm{~K}$ for all the samples. The MWCNT-reinforced samples exhibited a negative temperature coefficient of resistance (NTCR) behaviour where the resistivity of the samples decreases as a function of temperature. At higher temperatures, the resistivity exhibited temperature independent behaviour varying the value of resistivity from one another.

The addition of MWCNTs enhances the mobility of charge concentration compared with pure BZT and BCZT samples. We suppose that this difference is mainly due to the position of MWCNTs in the microstructure, i.e., in the grain boundary of the bulk ceramics. From the micrographs, we believe that the MWCNTs existing in the grain boundary will connect with one another to form a conductive network to transport electrons even at a very low-content. The aspect ratio of the MWCNTs also plays a vital role in reducing the $\rho_{\mathrm{dc}}$, as this helps to improve the interconnection. The increase in $\rho_{\mathrm{dc}}$ from $20 \times 10^{6}$ to $43 \times 10^{6} \Omega \mathrm{m}$ for BCZT10 and $121 \times 10^{6} \Omega \mathrm{m}$ at room temperature was mainly due to the amount of cerium present in the BZT. However, the detailed understanding of the electrical properties of MWCNT-reinforced ceramics is a big challenge and needs more work. In any case, the composition, element distribution and microstructures are the predominant factors to determine the electrical properties of CNT embedded ceramics.

The TEP measurements of the MWCNT embedded in BZT, BCZT10 and BCZT20 samples are shown in figure 4. The
$S$ values of CNT-embedded ceramics were negative in the whole temperature range studied. This indicates that the electrons are the majority charge carriers in all the samples. Also, the magnitude of $S$ was more compared to BZT and BCZT samples indicating MWCNT-reinforced ceramic samples as better thermoelectric materials. The value of $S$ is high at lower temperature and tends towards nearly zero with an increase in temperature and becomes constant, which is due to the solid-state reaction between the MWCNTs and ceramics. The absolute value of $S$ slightly decreases for MWCNT embedded-BCZT10 and BCZT20 compared to BZT. Here, the rare earth ion substitution widens the conduction band through hopping of charge carriers. The $S$ value is in good agreement with that of the non-degenerated semiconductor in the higher temperature ranges above $500 \mathrm{~K}$, as evidenced in dc electrical resistivity measurements.

\section{Conclusions}

MWCNT-reinforced pure and cerium-substituted BZT samples were prepared by the solid state reaction method. The SEM images show the formation of MWCNTs and MWCNTreinforced ceramics samples. The temperature dependent resistivity measurement exhibited a decrease in resistivity as a function of temperature indicating the NTCR behaviour of the reinforced ceramics. The decrease in the resistivity suggests the combined influence of MWCNTs and oxygen vacancies/ions or defects within cerium-substituted-BZT responsible for the long range motion of charge carriers. The Seebeck coefficient measurement revealed that all the samples are n-type non-degenerated semiconductors and the electrons are the major charge carriers. These findings also suggested that MWCNT-reinforced BCZT samples are promising candidates for n-type thermoelectric oxides compared to pure samples.

\section{Acknowledgements}

Thanks for the Department of Science and Technology (DST) New Delhi, India, for granting INSPIRE Fellowship vide No. DST/INSPIRE Fellowship/2011 dated 29 June 2011 to one of the authors (RS). The author (RS) is also thankful to the Vision Group of Science and Technology (VGST), Dept. of IT, BT and S\&T, Govt. of Karnataka, Bangalore for sanctioning research grant under CESEM project vide GRD No. 221 dated 24 January 2014.

\section{References}

[1] Treacy M M J, Ebbesen T W and Gibson J M 1996 Nature 381 678

[2] Wei B Q, Vajtai R and Ajayan P M 2001 Appl. Phys. Lett. 79 1172 
[3] Che J W, Cagin T and Goddard W A 2000 Nanotechnology 11 65

[4] Zhou W, Ooi Y H, Russo R, Papanek P, Luzzi D E and Fischer J E 2001 Chem. Phys. Lett. 3506

[5] Hennings D and Schnell A 1982 J. Am. Ceram. Soc. 65539

[6] Neirman S M 1988 J. Mater. Sci. 233973

[7] Ang Z Y C, Guo R and Bhalla A S 2002 J. Appl. Phys. 922655

[8] Halder S, Schneller T, Bottger U and Waser R 2005 Appl. Phys. A 8125

[9] Moura F, Simoes A Z, Stojanovic B D, Zaghete M A, Longo E and Varela J A 2008 J. Alloys Compds. 462129

[10] Salvetat J P, Kulik A J, Bonard J M, Briggs G A D, Stockli T and Metenier K 1999 Adv. Mater. 11161

[11] Huang Q and Gao L 2004 J. Mater. Chem. 142536

[12] Martin M, Jaroslav S, Milan P, Monika M and Dusan G 2014 Ceram. Int. 401289

[13] Kaleem A and Wei P 2008 Ceram. Eng. Sci. Proc. 2949

[14] Kumari L, Zhang T, Du G H, Li W Z, Wang Q W, Datye A et al 2009 Ceram. Int. 351775

[15] Connor M T, Roy S, Ezquerra T A and Balta Calleja F J 1998 Phys. Rev. B $\mathbf{5 7} 2286$

[16] Sui-Lin S and Ji L 2006 J. Am. Ceram. Soc. 893533
[17] Fan Y, Wang L, Li J, Li J, Sun S, Chen F et al 2010 Carbon 48 1743

[18] Liu Z Y, Xiao B L, Wang W G and Ma Z Y 2013 Carbon 62 35

[19] Maa P, Siddiquia N A, Maromb G and Kim J 2010 Composites: A 411345

[20] Sagar R, Madolappa S, Sharanappa N and Raibagkar R L 2013 Mater. Chem. Phys. 140119

[21] Sagar R and Raibagkar R L 2013 J. Alloys Compds. 549206

[22] Sagar R, Hudge P, Madolappa S, Kumbharkhane A C and Raibagkar R L 2012 J. Alloys Compds. 537197

[23] Sagar R, Madolappa S and Raibagkar R L 2012 Solid State Sci. 14211

[24] Sagar R, Madolappa S and Raibagkar R L 2011 Solid State Commun. 1511949

[25] Sagar R, Madolappa S and Raibagkar R L 2011 Ferroelectr. Lett. 38128

[26] Sagar R, Madolappa S and Raibagkar R L 2011 Integr. Ferroelectr. 13021

[27] Manafi S, Nadali H and Irani H R 2008 Mater. Lett. 624175

[28] Mazaheri M, Mari D, Hesabi Z R, Schaller R and Fantozzi G 2011 Compos. Sci. Technol. 72939 